\title{
Ossification of the ligamentum flavum in the upper cervical spine: A report of two cases and literature review
}

\author{
RUI HE and HUANG FANG \\ Department of Orthopedics, The First Affiliated Hospital of \\ the University of Science and Technology of China, Hefei, Anhui 230001, P.R. China
}

Received August 20, 2019; Accepted April 29, 2020

DOI: $10.3892 /$ etm.2020.8834

\begin{abstract}
Ossification of the ligamentum flavum (OLF) is rarely observed in the cervical spine, particularly not in the upper cervical spine. The present report describes two cases of OLF combined with cervical myelopathy and provides a literature review with a comparative analysis of previous studies and the present cases. Pre-operative CT, MRI and histological examination were performed to confirm the diagnosis of these patients (A 37-year-old female and 63-year-old male). In both cases, histological examination of surgical specimens revealed OLF. The symptoms of the two patients significantly improved after surgery and no recurrence was observed at 13 months after surgery. Furthermore, the clinical outcomes were satisfactory and the internal fixation was solid in the two cases. Review of the literature suggested that further research is required to reveal the optimal treatment methods for upper cervical OLF.
\end{abstract}

\section{Introduction}

Ossification of the ligamentum flavum (OLF) frequently occurs in the thoracic spine or at the junction of the thoracic and lumbar spine, but rarely in the cervical spine, particularly in the upper cervical spine (1-3). Miyazawa and Akiyama (4) previously reported on 50 cases of OLF of the cervical spine. However, OLF at the C1-2 level of the cervical spine is uncommon (4). A study reported on two cases of cervical myelopathy resulting from combined OLF and posterior longitudinal ligament (5). The present case study describes two cases of cervical myelopathy resulting from OLF alone. To the best of our knowledge, no similar cases have been reported in the English literature.

Correspondence to: Dr Huang Fang, Department of Orthopedics, The First Affiliated Hospital of the University of Science and Technology of China, 17 Lujiang Road, Hefei, Anhui 230001, P.R. China E-mail: 465330338@qq.com

Key words: upper cervical spine, ossification of ligamentum flavum, case report

\section{Case reports}

Case 1. A 37-year-old female presented with limb numbness and gradually progressive gait disturbance and clumsiness for 1 year. After 7 months, the patient fell and was subsequently not able to walk independently. The patient consulted a clinic accompanied by her husband and was then referred to the Department of Orthopedics of the First Affiliated Hospital of the University of Science and Technology of China (Hefei, China). On admission (March 2018), the patient exhibited gait disturbance due to cervical myelopathy without any fall or injury. Her grip strength was also decreased. Tests revealed elevated bilateral deep tendon reflexes of the limbs. Bilateral pathological reflexes were positive and bilateral Hoffmann's sign was positive. Motor weakness was noted mainly on the right side at and below the level of the deltoid. Sensory tests revealed significant hypoalgesia at and below the C6 level. The Japanese Orthopedic Association (JOA) score was 6 out of 17 (6). T1- and T2-weighted MRI revealed severe spinal cord compression at the $\mathrm{C} 1-2$ level. In the sagittal view, the dural sac was compressed from the posterior side by a hypointense mass (Fig. 1). CT indicated OLF at the C1-2 level (Fig. 2). A C1-2 laminectomy and OLF resection were performed (Fig. 3). H\&E staining of the resected ligamentum flavum was performed as previously described (7). Glass slides holding the paraffin sections were placed in staining racks. The paraffin was cleared from the samples with three 2-minchanges of xylene. Samples were then hydrated and stained in hematoxylin solution for $3 \mathrm{~min}$. Subsequently, the samples were stained with working eosin Y solution for $2 \mathrm{~min}$ and dehydrated. Finally, the slides were viewed using a microscope. The post-operative histological examination of surgical specimens revealed osseous tissues and enchondral ossification within the ligament, which confirmed the diagnosis of OLF (Fig. 4). At 1 month after the operation, the patient was able to walk independently. The imaging materials demonstrated that the internal fixation was successful and that the spinal cord was decompressed at the C1-2 level (Fig. 5). The post-operative JOA score was also improved to 11 , and this further improved to 12.5 at the 3-month follow-up. In addition, the patient's JOA score was still 12 at the 13-month follow-up.

Case 2. A 63-year-old male presented with sensory disturbance in the right upper extremity for 3 years, aggravated with weakness in lower extremities for 3 months. Recently 

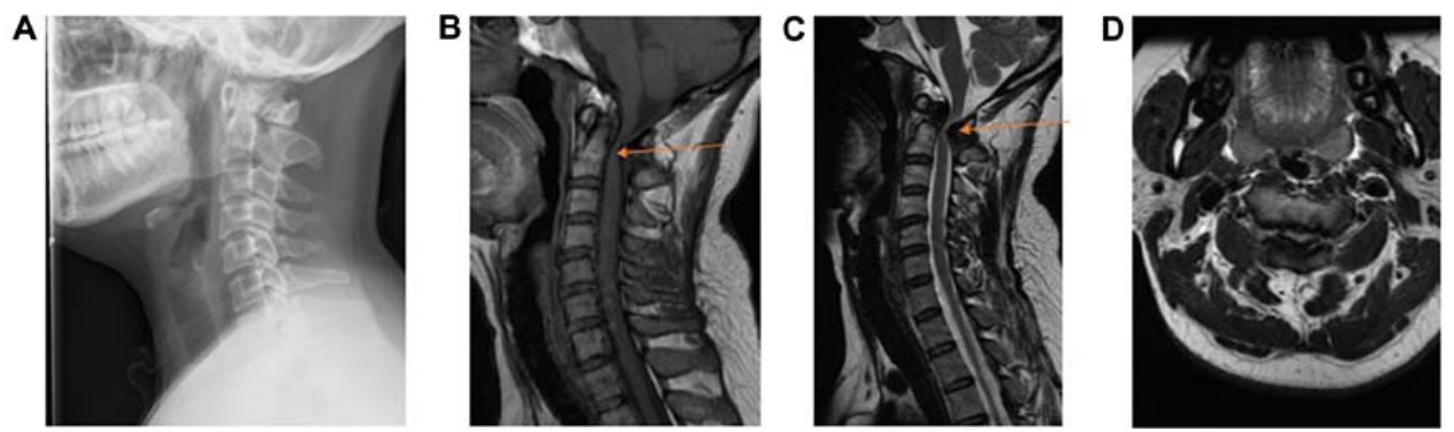

Figure 1. Case 1: (A) X-ray demonstrating the cervical curvature. MRI images demonstrating a (B) mass of isointensity on T1-weighted imaging and (C) hypointensity on T2-weighted imaging of the compressed dorsal spinal cord at the C1-2 level on sagittal view. The arrows indicate the lesion within the image. A T2-weighted axial image (D) demonstrating a hypointense mass at the C1-2 level causing spinal cord compression from laminae.
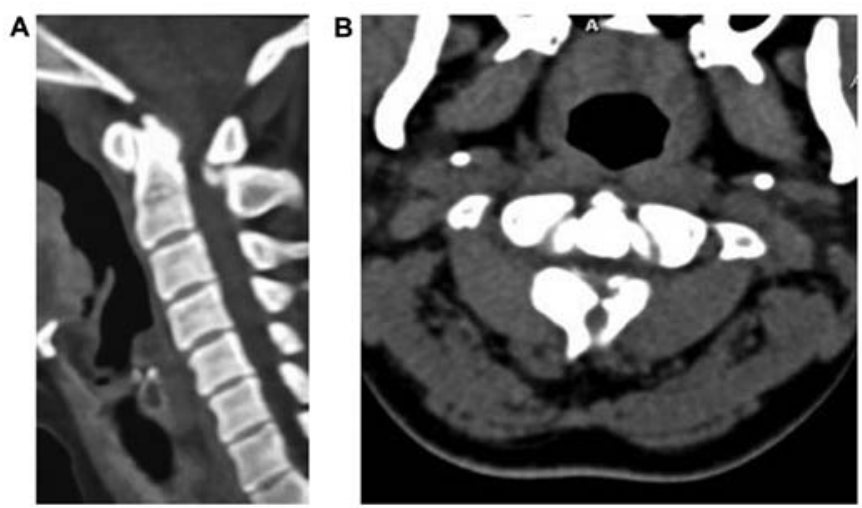

Figure 2. Case 2: (A) Sagittal and (B) axial CT scans demonstrating ossification of ligamentum flavum at the $\mathrm{C} 1-2$ level.

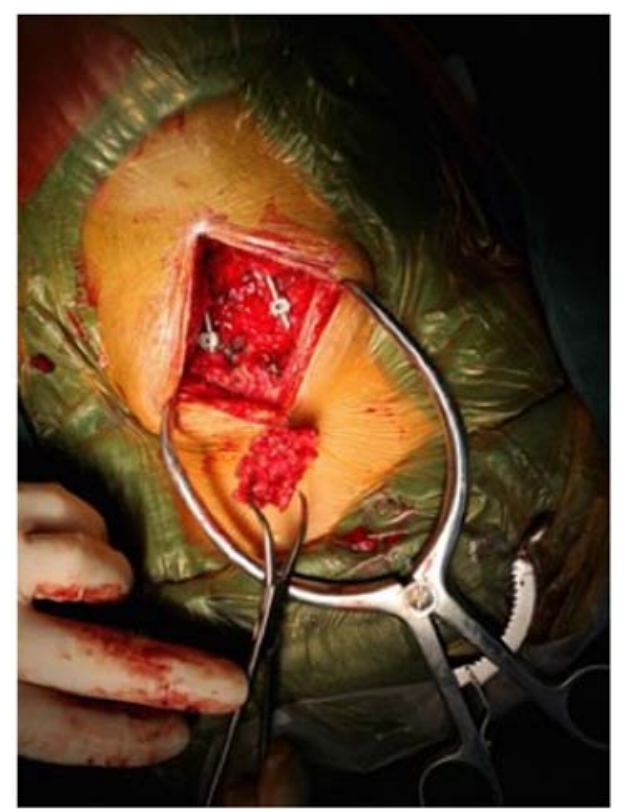

Figure 3. Case 1: En bloc resection of C1-2 laminae and operation of the lesion.

(March 2018), he had experienced difficulties with fine finger activities. After consulting a clinic due to concern about his symptoms, the patient was admitted to the Department of Orthopedics of the First Affiliated Hospital of the University

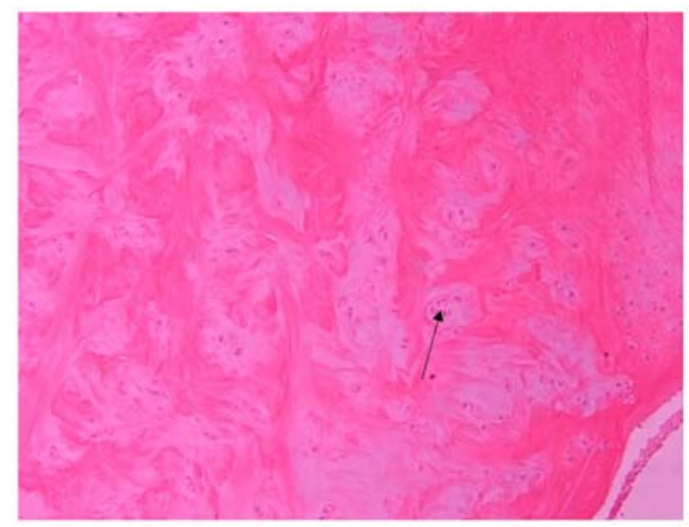

Figure 4. Case 1: Histological examination of the surgical specimens revealed osseous tissues (as indicated by the black arrow) and enchondral ossification within the ligament. Hematoxylin and eosin stain. Magnification, x40.
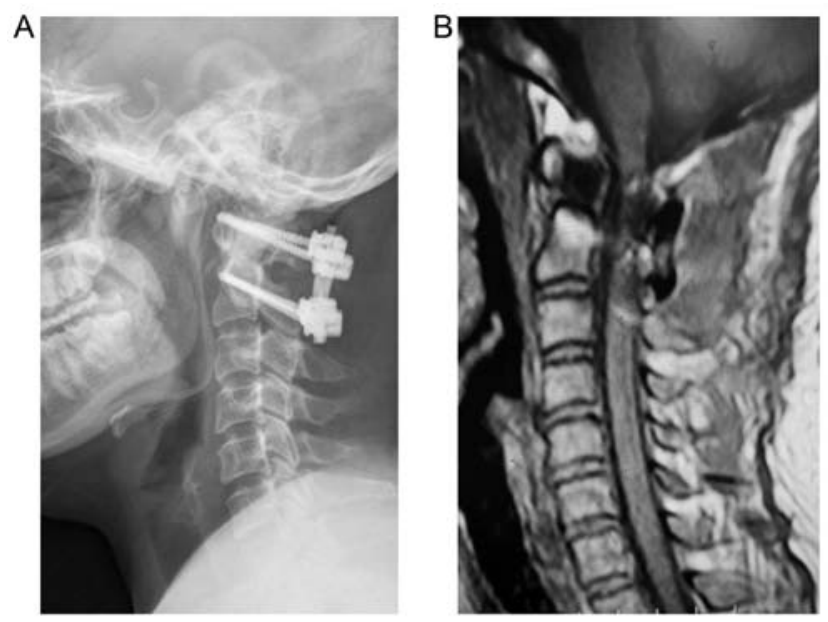

Figure 5. Case 1: (A) X-ray demonstrating that the internal fixation was successful, and (B) MRI revealing that the spinal cord was decompressed at the C1-2 level.

of Science and Technology of China (Hefei, China). Physical examination revealed a spastic gait and decreased bilateral grip, with decreased lower-extremity muscle strength. The deep tendon reflexes were elevated at the level of the biceps and triceps. The bilateral Hoffmann's sign and pathological reflexes in the lower extremities were positive. Sensory tests indicated hypoalgesia at and below the level of C5. The JOA score was 

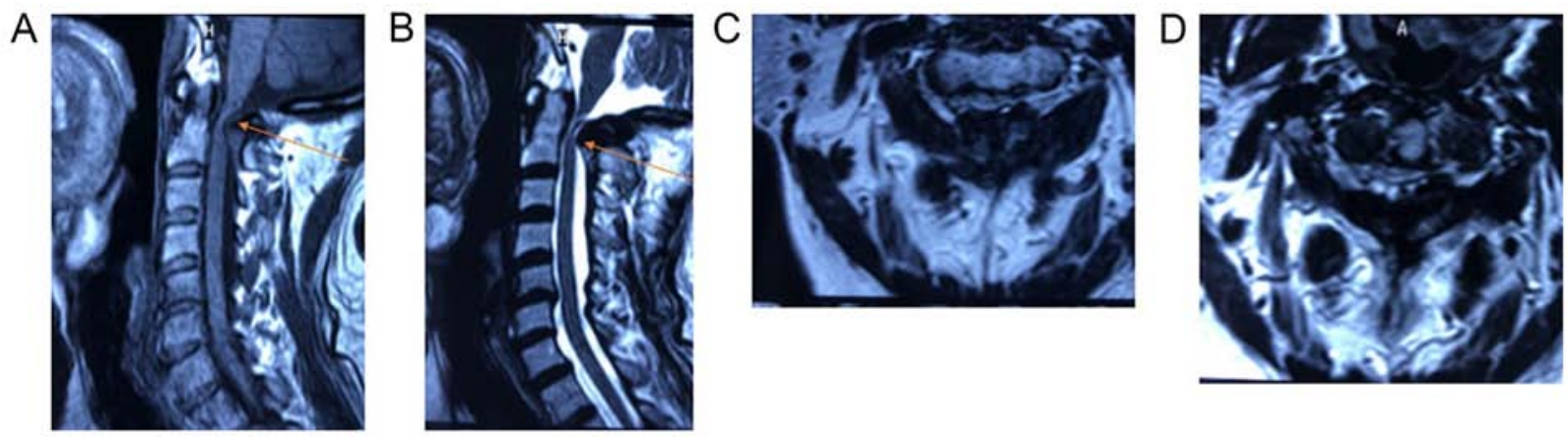

Figure 6. Case 2: Scanning images demonstrating isointensity with the spinal cord on (A) T1-weighted imaging and (B) hypointensity on the T2-weighted image posterior to the spinal cord at the C1-2 level on the sagittal view. (C) T2-weighted axial image demonstrating a hypointense mass at the C1-2 level causing spinal cord compression from laminae. (D) The spinal canal was significantly narrowed as viewed in a T2-weighted axial image at the C1-2 level.
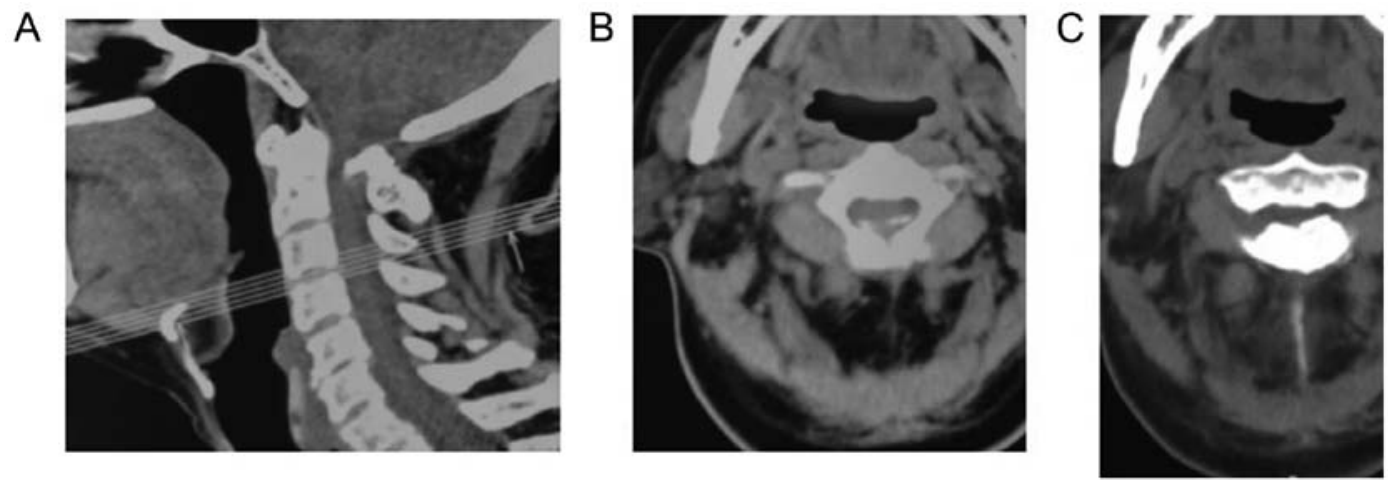

Figure 7. Case 2: (A) Sagittal and (B) axial CT scans demonstrating ossification of the ligamentum flavum at the C1-2 level. (C) The spinal canal was significantly narrowed in axial CT scans at C1-2 level.

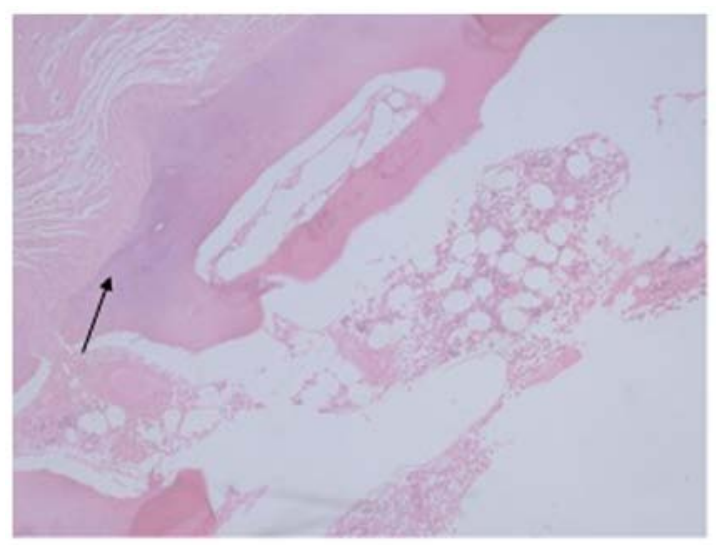

Figure 8. Case 2: Histological examination of the surgical specimens demonstrated (as indicated by the black arrow) osseous tissue and ossification within the ligamentum flavum. Hematoxylin and eosin stain. Magnification, x40.

6 out of 17.T1- and T2-weighted MRI revealed a hypointense mass compressing the dural sac from the posterior at $\mathrm{C} 1-2$ in the sagittal view and from the bilateral side of the lamina in the axial view (Fig. 6). Cervical dislocation at the level of C5-6 was also noted. No dural sac compression from the anterior aspect was noted in the cervical spine. CT revealed OLF at the C1-C2 level in the sagittal view and from the bilateral sides of the lamina in the axial view (Fig. 7). A C1-2 laminectomy and OLF resection were performed. A deformity in the posterior arch of the atlas was present, making it difficult to fix screws during the operation. Therefore, occipitocervical fusion and internal fixation were adopted in this case. H\&E staining was performed as described above. After the surgery, histological examination of surgical specimens revealed osseous tissue and ossification within the ligamentum flavum, which confirmed the diagnosis of OLF in the upper cervical spine (Fig. 8). MRI scans revealed that the spinal cord was decompressed at the C1-2 level and that the OLF was totally resected (Fig. 9). The patient's symptoms significantly improved at one week after the operation and his JOA score improved to 12 at the 3-month follow-up. In addition, the patient's JOA score was still 12 at the 13-month follow-up.

\section{Discussion}

The first report of a case of OLF was in the 1920s (8). Thereafter, numerous cases were reported in Asian countries (1). Liang et al (9) determined that the prevalence of cervical OLF (C-OLF) was $0.3 \%$ and there was no significant difference in the prevalence of C-OLF between males and females in the Chinese population. Symptomatic OLF has predominantly been reported in the lower thoracic spine (10-12). However, symptomatic OLF in the cervical spine is rarely observed (13). OLF tends to arise from the lateral capsular portion of the ligamentum flavum, whereas OLF characteristically occurs in the thoracic spine of older females $(14,15)$. Miyazawa and Akiyama (4) reviewed 50 cases of OLF in the cervical spine that were reported between 1962 and 2005. The mean age of the 

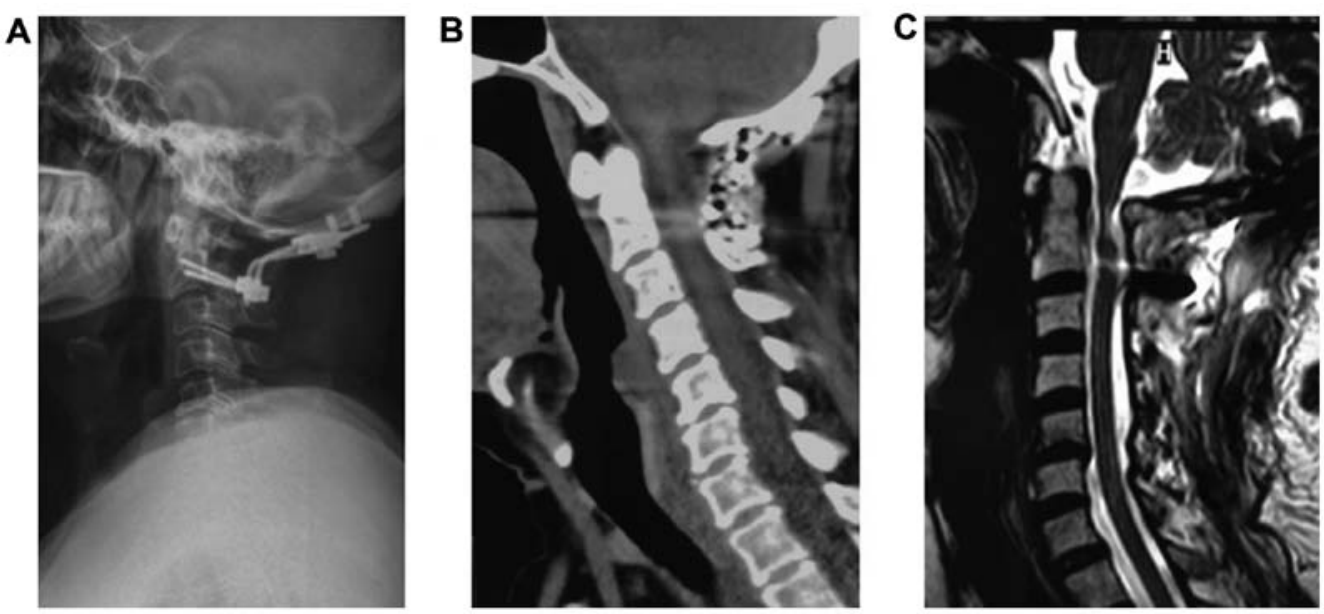

Figure 9. Case 2: (A) X-ray demonstrating that the internal fixation was successful. (B) CT scan showing that the ligamentum flavum was resected. (C) MRI image showing that the spinal cord was decompressed at the C1-2 level.

patients was 56.3 years, with a male/female ratio of $1.44: 1(16)$ Sensorimotor deficits were common in those patients with OLF. Sensory disturbance in the extremities is usually the initial symptom of OLF (4). Rahimizadeh et al (17) reviewed 68 cases of C-OLF resulting in progressive quadriparesis and demonstrated that $\mathrm{C}$-OLF rarely leads to cervical myeloradiculopathy. Different imaging techniques have different advantages in the diagnosis of disease (18). MRI may reveal hypertrophy of the ligamentum flavum and compression of the spinal cord in the cervical spine (18). The causes of OLF in the cervical spine remain to be elucidated (19). The diagnosis of OLF is thought to be different from that of other pathologies in that the clinician must distinguish OLF from other pathologies including spinal tumors (19). Based on CT scans, bone destruction may at times be observed. The lesion maybe easily detected on MRI. The long axis of the tumor is parallel to the long axis of the spinal cord. As the disease progresses, cystic changes and spinal cavities are prone to occur. Regarding chondroma in the ligamentum flavum or the laminar arch, $\mathrm{X}$-rays may display round or irregular bone-like density shadows adjacent to the vertebral body. The internal density was uneven, the lesions were clear and side attachment was involved in both of the reported cases. MRI, CT and myelography may be used to assess the size and extent of the damage caused by osteochondroma and reveal the structures obscured by ordinary X-rays. CT is advantageous compared with MRI for the observation of osteochondroma, and is able to reveal details of the cartilage and bones, whereas MRI displays the nerve structure more clearly than CT. However, the diagnosis must rely on pathological biopsy. Post-operative pathology also proved the diagnosis of C-OLF. According to several studies, the characteristics of C-OLF are similar to those observed in thoracic OLF. Yayama et al (20) reported that certain metabolic disorders may promote the ossification of ligaments and that specific osteogenic cytokines, including bone morphogenetic protein 2 and transforming growth factor- $\beta$, may have a role in the development of ossification. The optimal treatment for C-OLF remains to be determined, since only a small number of clinical reports are currently available (21). However, for patients with apparent or progressive neurological deficits, lesional resection and decompression of the cervical spinal cord are usually the preferred treatment options (22). Various tools (such as clam, ultrasonic osteotome and rongeur) may be used to remove the lesion. The actual tools used depend on the facilities at the local hospital, the surgeon's proficiency and the financial ability of the patients (22). During the surgeries of the two cases in the present report, enlarged and hardened ligamentum flavum were observed. The white powder-like substance inside the ligament was solid. The dorsal side of the cervical spinal cord was compressed. In the two patients of the present study, the surgeries were performed with the aid of an ultrasonic osteotome. The ultrasonic osteotome has the advantage of tissue selectivity and eliminates the dangerous rotatory motion of the high speed burr, thus allowing a more safe removal of C-OLF (23). In the present study, since the lesion was located at the junction between the occipital bone and the neck, once the laminectomy was performed, cervical instability was likely to occur (24). Therefore, fusion surgery was selected for these patients rather than stand-alone decompressive laminectomy. A search of the English literature revealed the importance of decompression, but few studies mentioned the method of internal fixation $(25,26)$. It was hypothesized that the process of decompression may partially destroy the stability of the cervical spine. There are certain difficulties in performing the process of internal fixation in the upper cervical spine. The experience of the surgeon, deformity of the upper cervical spine and facilities of local hospitals may ultimately influence the choice of modality of internal fixation. Further research is required to develop guidelines for treating these diseases. During surgery, spinal cord injury and cerebrospinal fluid leakage may occur due to dural adhesion (27). To ensure the safety of the operation, it may be recommended that only doctors with specific experience in upper cervical surgery perform these operations. A longer follow-up period may further confirm the diagnosis and therapy of these cases found in the present report. The patients were actively followed up for 18 months and it was determined that their clinical symptoms did not recur. However, due to economic reasons, the patients did not receive a complete imaging assessment.

In conclusion, the present study described two cases of neurological deficits resulting from C-OLF alone. The symptoms were significantly improved in both patients following laminectomy 
and internal fixation. On the basis of the literature review, further research is required to reveal optimal treatment for C-OLF.

\section{Acknowledgements}

Not applicable.

\section{Funding}

No funding was received.

\section{Availability of data and materials}

The datasets used and/or analyzed during the current study are available from the corresponding author on reasonable request.

\section{Authors' contributions}

RH and HF examined patients and collected clinical data; $\mathrm{RH}$ performed the surgery and analyzed radiological imaging data; RH and HF wrote the manuscript. All authors read and approved the final manuscript.

\section{Ethics approval and consent to participate}

Informed consent was obtained from the study subjects and the study followed the institute's committee on human research protocols.

\section{Patient consent for publication}

Informed written consent was obtained from the patients prior to all procedures described in the report, as well as for the use of the patient's clinical information and images for published scientific purposes.

\section{Competing interests}

The authors declare that they have no competing interests.

\section{References}

1. Aizawa T, Sato T, Sasaki H, Kusakabe T, Morozumi N and Kokubun S: Thoracic myelopathy caused by ossification of the ligamentum flavum: Clinical features and surgical results in the Japanese population. J Neurosurg Spine 5: 514-519, 2006.

2. Ben Hamouda K, Jemel H, Haouet S and Khaldi M: Thoracic myelopathy caused by ossification of the ligamentum flavum: A report of 18 cases. J Neurosurg 99 (Suppl 2): 157-161, 2003.

3. Chou YC, Lee CC, Yen PS, Lin JF, Su CF, Lin SZ and Chen WF: Cough induced by ossification of the ligamentum flavum in the high cervical spine. Case report. J Neurosurg 100 (Suppl 4): 364-366, 2004.

4. Miyazawa $\mathrm{N}$ and Akiyama I: Ossification of the ligamentum flavum of the cervical spine. J Neurosurg Sci 51: 139-144, 2007.

5. Kotani Y, Takahata M, Abumi K, Ito M, Sudo H and Minami A: Cervical myelopathy resulting from combined ossification of the ligamentum flavum and posterior longitudinal ligament: report of two cases and literature review. Spine J 13: e1-6, 2013.

6. Gupte G, Peters CM, Buchowski JM, Zebala LP, Sudo H and Minami A: Reliability of the Neck Disability Index and Japanese Orthopedic Association questionnaires in adult cervical radiculopathy and myelopathy patients when administered by telephone or via online format. Spine J 19: 1154-1161, 2019.

7. Cardiff RD, Miller CH and Munn RJ: Manual hematoxylin and eosin staining of mouse tissue section. Cold Spring Harb Protoc 2014: 655-658, 2014.
8. Polgar F: Über interarkuelle Wirbelverkalkung. Fortschr Geb Rontgen 40: 292-298, 1929 (In German).

9. Liang H, Liu G, Lu S, Chen S, Jiang D, Shi H and Fei Q: Epidemiology of ossification of the spinal ligaments and associated factors in the Chinese population: A cross-sectional study of 2000 consecutive individuals. BMC Musculoskelet Disord 20: 253, 2019.

10. Ando K, Imagama S, Kobayashi K, Hida T, Ito K, Tsushima M, Ishikawa $\mathrm{Y}$, Matsumoto A, Nishida $\mathrm{Y}$ and Ishiguro N: Comparative study of surgical treatment and nonsurgical follow up for thoracic ossification of the posterior longitudinal ligament: radiological and clinical evaluation. Spine 42: 407-410, 2017.

11. Singhal U, Jain M, Jaiswal AK and Behari S: Unilateral ossified ligamentum flavum in the high cervical spine causing myelopathy. Indian J Orthop 43: 305-308, 2009.

12. Christiano LD, Assina R and Goldstein IM: Ossification of the ligamentum flavum: A unique report of a Hispanic woman. Neurosurg Focus 30: E15, 2011.

13. Kawaguchi Y, Nakano M, Yasuda T, Seki S, Hori T, Suzuki K, Makino $\mathrm{H}$ and Kimura T: Characteristics of ossification of the spinal ligament; incidence of ossification of the ligamentum flavum in patients with cervical ossification of the posterior longitudinal ligament - Analysis of the whole spine using multidetector CT. J Orthop Sci 21: 439-445, 2016.

14. Miyasaka K, Kaneda K, Sato S, Iwasaki Y, Abe S, Takei H, Tsuru M, Tashiro K, Abe $\mathrm{H}$ and Fujioka Y: Myelopathy due to ossification or calcification of the ligamentum flavum: Radiologic and histologic evaluations. AJNR Am J Neuroradiol 4: 629-632, 1983.

15. Takahashi T, Hanakita J and Minami M: Pathophysiology of calcification and ossification of the ligamentum flavum in the cervical spine. Neurosurg Clin N Am 29: 47-54, 2018.

16. Ohara Y: Ossification of the ligaments in the cervical spine, including ossification of the anterior longitudinal ligament, ossification of the posterior longitudinal ligament, and ossification of the ligamentum flavum. Neurosurg Clin N Am 29: 63-68, 2018.

17. Rahimizadeh A, Asgari N, Soufiani H and Rahimizadeh S: Ossification of the cervical ligamentum flavum and case report with myelopathy. Surg Neurol Int 9: 263, 2018.

18. Ponte A, Orlando G and Siccardi GL: The true ponte osteotomy: By the one who developed it. Spine Deform 6: 2-11, 2018.

19. Muthukumar N and Muthukumar N: Ossification of the ligamentum flavum as a result of fluorosis causing myelopathy: Report of two cases. Neurosurgery 56: E622, 2005.

20. Yayama T, Uchida K, Kobayashi S, Kokubo Y, Sato R, Nakajima H, Takamura T, Bangirana A, Itoh $\mathrm{H}$ and Baba $\mathrm{H}$ : Thoracic ossification of the human ligamentum flavum: Histopathological and immunohistochemical findings around the ossified lesion. J Neurosurg Spine 7: 184-193, 2007.

21. Chen Y, Chen DY, Wang XW, Lu XH, Yang HS and Miao JH: Single-stage combined decompression for patients with tandem ossification in the cervical and thoracic spine. Arch Orthop Trauma Surg 132: 1219-1226, 2012.

22. Liang H, Liu G, Lu S, Chen S, Jiang D, Shi H and Fei Q: Epidemiology of ossification of the spinal ligaments and associated factors in the Chinese population: a cross-sectional study of 2000 consecutive individuals. BMC Musculoskelet Disord 20: 253, 2019.

23. Kim CH, Renaldo N, Chung CK and Lee HS: Use of an ultrasonic osteotome for direct removal of beak-type ossification of posterior longitudinal ligament in the thoracic spine. J Korean Neurosurg Soc 58: 571-577, 2015.

24. Li J, Yan DL, Gao LB, Tan PX, Zhang ZH and Zhang Z: Comparison percutaneous cervical disc nucleoplasty and cervical discectomy for the treatment of cervical disc herniation. Zhonghua Wai Ke Za Zhi 44: 822-825, 2006 (In Chinese).

25. An B, Li XC, Zhou CP, Wang BS, Gao HR, Ma HJ, He Y, Zhou HG, Yang HJ and Qian JX: Percutaneous full endoscopic posterior decompression of thoracic myelopathy caused by ossification of the ligamentum flavum. Eur Spine J 28: 492-501, 2019.

26. Ikuta K, Tarukado K, Senba H, Kitamura T, Komiya N, Fukutoku Y and Shidahara S: Decompression procedure using a microendoscopic technique for thoracic myelopathy caused by ossification of the ligamentum flavum. Minim Invasive Neurosurg 54: 271-273, 2011.

27. Yang H, Lu X, Wang X, Chen D, Yuan W, Yang L and Liu Y: A new method to determine whether ossified posterior longitudinal ligament can be resected completely and safely: Spinal canal 'Rule of Nine' on axial computed tomography. Eur Spine J 24: 1673-1680, 2015.

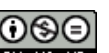

This work is licensed under a Creative Commons Attribution-NonCommercial-NoDerivatives 4.0 International (CC BY-NC-ND 4.0) License. 